\title{
PHASE PORTRAITS OF QUADRATIC LOTKA-VOLTERRA SYSTEMS WITH A DARBOUX INVARIANT IN THE POINCARÉ DISC
}

\author{
YUDY BOLAÑOS ${ }^{1}$, JAUME LLIBRE $^{1}$ AND CLAUDIA VALLS ${ }^{2}$
}

\begin{abstract}
We characterize the global phase portraits in the Poincaré disc of all the planar Lotka-Volterra quadratic polynomial differential systems having a Darboux invariant.
\end{abstract}

\section{INTRODUCTION AND STATEMENT OF MAIN RESUltS}

Let $\mathbb{R}[x, y]$ (resp. $\mathbb{C}[x, y]$ ) be the ring of the polynomials in the variables $x$ and $y$ with coefficients in $\mathbb{R}$ (resp. $\mathbb{C}$ ). We consider a system of polynomial differential equations or simply a polynomial differential system in $\mathbb{R}^{2}$ defined by

$$
\dot{x}=P(x, y), \quad \dot{y}=Q(x, y),
$$

where $P, Q \in \mathbb{R}[x, y]$ and the dot denotes derivative with respect to the independent variable $t$ usually called the time. We say that the maximum of the degrees of the polynomials $P$ and $Q$ is the degree of system (1). Usually a quadratic polynomial differential system of degree 2 is called simply a quadratic system.

Sometimes we shall talk about the quadratic vector field

$$
\mathcal{X}=P(x, y) \frac{\partial}{\partial x}+Q(x, y) \frac{\partial}{\partial y} .
$$

associated to the quadratic system (1). A non-locally constant real function $H$ of class $C^{1}$ defined on an open set $U$ is a first integral of the polynomial vector field $\mathcal{X}$ if $H(x(t), y(t))$ is constant for all value of $t$ for which the solution $(x(t), y(t))$ of $\mathcal{X}$ is defined on $U$. We observe that $H$ is a first integral of $\mathcal{X}$ if and only if $\mathcal{X} H=0$ on $U$.

Let $f \in \mathbb{C}[x, y] \backslash\{0\}$. The algebraic curve $f(x, y)=0$ is an invariant algebraic curve of the polynomial system (1) if for some polynomial $k \in \mathbb{C}[x, y]$ we have

$$
\mathcal{X} f=P \frac{\partial f}{\partial x}+Q \frac{\partial f}{\partial y}=k f
$$

The polynomial $k$ is called the cofactor of the invariant algebraic curve $f=0$.

Let $g, h \in \mathbb{C}[x, y]$ and assume that $g$ and $h$ are relatively prime in the ring $\mathbb{C}[x, y]$ or that $h=1$. Then the function $\exp (g / h)$ is called an exponential factor of system (1) if for some polynomial $k \in \mathbb{C}[x, y]$ of degree at most 1 we have that

$$
\mathcal{X}\left(\exp \left(\frac{g}{h}\right)\right)=k \exp \left(\frac{g}{h}\right) .
$$

2010 Mathematics Subject Classification. 34A34, 34C05, 34C14.

Key words and phrases. Phase portrait, quadratic Lotka-Volterra systems, Darboux invariant, Poincaré compactification, Poincaré disc. 
A non-constant function $I=I(x, y, t)$ is an invariant of $\mathcal{X}$ if

$$
\frac{d I}{d t}=\frac{d I}{d x} \dot{x}+\frac{d I}{d y} \dot{y}+\frac{d I}{d t}=0 .
$$

That is, $I$ is an invariant of $\mathcal{X}$ if it is a first integral depending on the time $t$. Moreover we say that $I$ is a Darboux invariant if it is an invariant which can be written as

$$
I=f_{1}^{\lambda_{1}} \cdots f_{p}^{\lambda_{p}} F_{1}^{\mu_{1}} \cdots F_{q}^{\mu_{q}} \exp (s t)
$$

where $f_{1}=0, \ldots, f_{p}=0$ are invariant algebraic curves of $\mathcal{X}, F_{1}, \ldots, F_{q}$ are exponential factors of $\mathcal{X}, \lambda_{1}, \ldots, \lambda_{p}, \mu_{1}, \ldots, \mu_{q} \in \mathbb{C}$ and $s \in \mathbb{R} \backslash\{0\}$.

The quadratic Lotka-Volterra systems are of the form

$$
\dot{x}=x(a x+b y+c), \quad \dot{y}=y(A x+B y+C),
$$

where $a, b, c, A, B, C$ are constants. In this paper we study the quadratic systems (3) that possess a Darboux invariant.

System (3) and its generalization to dimension $n$, since were originally studied by Lotka and Volterra in [12, 18], are called Lotka-Volterra systems. Later on these differential systems were studied by Kolmogorov [9], and after his work some authors called them Kolmogorov systems. There are many natural phenomena that can be modeled by these differential systems such as the time evolution of conflicting species in biology [15], chemical reactions, plasma physics [10], hydrodynamics [2], economics,

The knowledge of a first integral is of special interest for a differential system in the plane because it allows to compute the explicit expressions of the trajectories of the system. However, when we cannot compute a first integral of the system it is interesting to know if the system has an invariant. Roughly speaking, with a first integral we can describe completely the phase portrait of a planar differential system, while with an invariant we only can describe its asymptotic behavior, i.e. the $\omega$-and $\alpha$-limit sets of its trajectories.

Our first result is the characterization of the quadratic Lotka-Volterra systems (3) having a Darboux invariant.

Theorem 1. The quadratic Lotka-Volterra systems (3) having a Darboux invariant are either

$$
\dot{x}=x(a x+b y+c), \quad \dot{y}=C y
$$

with $C \neq 0$ and $a^{2}+b^{2} \neq 0$, or

$$
\dot{x}=x\left(-A \frac{l_{2}}{l_{1}} x-B \frac{l_{2}}{l_{1}} y+c\right), \quad \dot{y}=y(A x+B y+C)
$$

with $l_{1} l_{2} \neq 0, c^{2}+C^{2} \neq 0$ and $A^{2}+B^{2} \neq 0$. Their Darboux invariants are $e^{-C t} y$ and $e^{\left(-c l_{1}-C l_{2}\right) t} x^{l_{1}} y^{l_{2}}$ respectively, where $l_{1}$ and $l_{2}$ are constants.

Theorem 1 will be proved in section 2. Theorem 1 improves some preliminary results on the Darboux invariants of the quadratic Lotka-Volterra systems given in Theorem 1 of [3].

Note that if $l_{2}=0$ then system (5) becomes system (4) after the change of variables and parameters $(x, y, A, B, C, c) \rightarrow(y, x, b, a, c, C)$. Hence, in system (5) we have excluded the case $l_{2}=0$. 
We remark that in Theorem 1 we do not consider the differential systems (4) and (5) which become linear differential systems, i.e. we only consider quadratic differential systems (4) and (5). This is obtained with the conditions $a^{2}+b^{2} \neq 0$ and $A^{2}+B^{2} \neq 0$ in the systems (4) and (5), respectively.

We observe that the conditions $C \neq 0$ and $c^{2}+C^{2} \neq 0$, in the systems (4) and (5) respectively, are necessary in order that such systems really have a Darboux invariant.

Our next results provide the global phase portraits of systems (4) and (5) in the Poincaré disc, see Theorems 2 and 3, respectively.

Theorem 2. The phase portrait of any quadratic Lotka-Volterra system (4) is topologically equivalent to one of the 7 configurations of Figures 1-4.

Theorem 3. The phase portrait of any quadratic Lotka-Volterra system (5) is topologically equivalent either to one of the 24 configurations of Figures 5-10, or to the configuration $b=0$ of Figure 2, or to the configuration $b=0$ of Figure 4.

In sections 5 and 6 we shall prove Theorems 2 and 3, respectively. But before that we recall the notions of the Poincaré compactification (section 3), the definitions of separatrices and of canonical regions (section 4) that we will need later on for drawing the global phase portraits of systems (4) and (5).

\section{Proof of TheOREM 1}

In order to prove Theorem 1 first we recall a result from the Darboux theory of integrability that we will use, for a proof see Theorem 8.7 of [6].

Proposition 4. Supposse that a polynomial system (1) of degree $n$ admits $p$ irreducible invariant algebraic curves $f_{i}=0$ with cofactors $k_{i}$ for $i=1, \ldots, p$, q exponential factors $F_{j}=\exp \left(g_{j} / h_{j}\right)$ with cofactors $l_{j}$ for $j=1, \ldots, q$. If there exist $\lambda_{i}, \mu_{j} \in \mathbb{C}$ not all zero such that

$$
\sum_{i=1}^{p} \lambda_{i} k_{i}+\sum_{j=1}^{q} \mu_{j} l_{j}=-s
$$

for some $s \in \mathbb{R} \backslash\{0\}$, then the (multi-valued) function

$$
f_{1}^{\lambda_{1}} \ldots f_{p}^{\lambda_{p}} F_{1}^{\mu_{1}} \cdots F_{q}^{\mu_{q}} \exp (s t)
$$

is a Darboux invariant of $\mathcal{X}$.

Proof of Theorem 1. It is easy to verify that the straight lines $x=0$ and $y=0$ are invariant algebraic curves of the quadratic Lotka-Volterra systems (3) with cofactors $k_{1}=a x+b y+c$ and $k_{2}=A x+B y+C$ respectively. So solving equation (6) with $k_{1}$ and $k_{2}$ we obtain two solutions which are either

$$
s=-C l_{2}, \quad A=0, \quad B=0, \quad l_{1}=0,
$$

or

$$
s=-c l_{1}-C l_{2}, \quad a=-A l_{2} / l_{1}, \quad b=-B l_{2} / l_{1}, \quad l_{1} \neq 0 .
$$

From (8) we arrive to the family of quadratic Lotka-Volterra systems (4) having the Darboux invariant $e^{-C t} y$ when $C \neq 0$. Similarly from (9) we obtain the family (5) with the Darboux invariant $\mathrm{e}^{-\left(c l_{1}+C l_{2}\right) t} x^{l_{1}} y^{l_{2}}$ when $-c l_{1}-C l_{2} \neq 0$, as it is stated in the theorem. 


\section{Poincaré COMPACTIFICATION}

Let $\mathcal{X}$ be a planar vector field of degree $n$. The Poincaré compactified vector field $p(\mathcal{X})$ corresponding to $\mathcal{X}$ is an analytic vector field induced on $\mathbb{S}^{2}$ as follows (see, for instance [8], or Chapter 5 of [6]). Let $\mathbb{S}^{2}=\left\{y=\left(y_{1}, y_{2}, y_{3}\right) \in \mathbb{R}^{3}: y_{1}^{2}+y_{2}^{2}+y_{3}^{2}=1\right\}$ (the Poincaré sphere) and $T_{y} \mathbb{S}^{2}$ be the tangent space to $\mathbb{S}^{2}$ at point $y$. Consider the central projection $f: T_{(0,0,1)} \mathbb{S}^{2} \rightarrow \mathbb{S}^{2}$. This map defines two copies of $\mathcal{X}$, one in the northern hemisphere and the other in the southern hemisphere. Denote by $\mathcal{X}^{\prime}$ the vector field $D f \circ \mathcal{X}$ defined on $\mathbb{S}^{2}$ except on its equator $\mathbb{S}^{1}=\left\{y \in \mathbb{S}^{2}: y_{3}=0\right\}$. Clearly $\mathbb{S}^{1}$ is identified to the infinity of $\mathbb{R}^{2}$. In order to extend $\mathcal{X}^{\prime}$ to a vector field on $\mathbb{S}^{2}$ (including $\mathbb{S}^{1}$ ) it is necessary that $\mathcal{X}$ satisfies suitable conditions. In the case that $\mathcal{X}$ is a planar vector field of degree $n$ then $p(\mathcal{X})$ is the only analytic extension of $y_{3}^{n-1} \mathcal{X}^{\prime}$ to $\mathbb{S}^{2}$. On $\mathbb{S}^{2} \backslash \mathbb{S}^{1}$ there are two symmetric copies of $\mathcal{X}$, and knowing the behavior of $p(\mathcal{X})$ around $\mathbb{S}^{1}$, we know the behavior of $\mathcal{X}$ at infinity. The projection of the closed northern hemisphere of $\mathbb{S}^{2}$ on $y_{3}=0$ under $\left(y_{1}, y_{2}, y_{3}\right) \longmapsto\left(y_{1}, y_{2}\right)$ is called the Poincaré disc, and it is denoted by $\mathbb{D}$. The Poincaré compactification has the property that $\mathbb{S}^{1}$ is invariant under the flow of $p(\mathcal{X})$.

In this paper we say that two polynomial vector fields $\mathcal{X}$ and $\mathcal{Y}$ on $\mathbb{R}^{2}$ are topologically equivalent if there exists a homeomorphism on $\mathbb{S}^{2}$ preserving the infinity $\mathbb{S}^{1}$ carrying orbits of the flow induced by $p(\mathcal{X})$ into orbits of the flow induced by $p(\mathcal{Y})$, preserving or reversing simultaneously the sense of all orbits.

As $\mathbb{S}^{2}$ is a differentiable manifold, for computing the expression for $p(\mathcal{X})$, we can consider the six local charts $U_{i}=\left\{y \in \mathbb{S}^{2}: y_{i}>0\right\}$, and $V_{i}=\left\{y \in \mathbb{S}^{2}: y_{i}<0\right\}$ where $i=1,2,3$; and the diffeomorphisms $F_{i}: U_{i} \rightarrow \mathbb{R}^{2}$ and $G_{i}: V_{i} \rightarrow \mathbb{R}^{2}$ for $i=1,2,3$ are the inverses of the central projections from the planes tangent at the points $(1,0,0),(-1,0,0),(0,1,0),(0,-1,0),(0,0,1)$ and $(0,0,-1)$ respectively. We denote by $(u, v)$ the value of $F_{i}(y)$ or $G_{i}(y)$ for any $i=1,2,3$ (so $(u, v)$ represents different things according to the local charts under consideration).

The expression for $p(\mathcal{X})$ in the local chart $\left(U_{1}, F_{1}\right)$ is given by

$$
\dot{u}=v^{n}\left[-u P\left(\frac{1}{v}, \frac{u}{v}\right)+Q\left(\frac{1}{v}, \frac{u}{v}\right)\right], \quad \dot{v}=-v^{n+1} P\left(\frac{1}{v}, \frac{u}{v}\right) .
$$

The expression for $p(\mathcal{X})$ in local chart $\left(U_{2}, F_{2}\right)$ is

$$
\dot{u}=v^{n}\left[P\left(\frac{u}{v}, \frac{1}{v}\right)-u Q\left(\frac{u}{v}, \frac{1}{v}\right)\right], \quad \dot{v}=-v^{n+1} Q\left(\frac{u}{v}, \frac{1}{v}\right),
$$

and for $\left(U_{3}, F_{3}\right)$ is

$$
\dot{u}=P(u, v), \quad \dot{v}=Q(u, v) .
$$

The expression for $p(\mathcal{X})$ in the charts $\left(V_{i}, G_{i}\right)$ is the same as for $\left(U_{i}, F_{i}\right)$ multiplied by $(-1)^{n-1}$ for $i=1,2,3$. The points of $\mathbb{S}^{1}$ in any chart have $v=0$. Thus we obtain a polynomial vector field in each local chart.

\section{SEPARATRices AND CANONICAL REGIONS}

Let $p(\mathcal{X})$ be the Poincaré compactification in the Poincare disc $\mathbb{D}$ of the polynomial differential system (1) defined in $\mathbb{R}^{2}$, and let $\Phi$ be its analytic flow. Following Markus [14] and Neumann [16] we denote by $(U, \Phi)$ the flow of a differential system on an invariant set $U \subset \mathbb{D}$ under the flow $\Phi$. The flow $(U, \Phi)$ is said to be parallel if it is topologically equivalent to one of the following flows: 
(i) The flow defined in $\mathbb{R}^{2}$ by the differential system $\dot{x}=1, \dot{y}=0$, called strip flow.

(ii) The flow defined in $\mathbb{R}^{2} \backslash\{\mathbf{0}\}$ by the differential system in polar coordinates $\dot{r}=0, \dot{\theta}=1$, called annular flow.

(iii) The flow defined in $\mathbb{R}^{2} \backslash\{\mathbf{0}\}$ by the differential system in polar coordinates $\dot{r}=r, \dot{\theta}=0$, called spiral or radial flow.

It is known that the separatrices of a polynomial vector field $p(\mathcal{X})$ in the Poincaré disc $\mathbb{D}$ are:

(I) all the orbits of $p(\mathcal{X})$ which are in the boundary $\mathbb{S}^{1}$ of the Poincaré disc (i.e. at the infinity of $\mathbb{R}^{2}$ ),

(II) all the finite singular points of $p(\mathcal{X})$,

(III) all the limit cycles of $p(\mathcal{X})$, and

(IV) all the separatrices of the hyperbolic sectors of the finite and infinite singular points of $p(\mathcal{X})$.

Let $\mathcal{S}$ be the union of the separatrices of the flow $(\mathbb{D}, \Phi)$ defined by $p(\mathcal{X})$ in the Poincaré disc $\mathbb{D}$. It is easy to check that $\mathcal{S}$ is an invariant closed set. If $N$ is a connected component of $\mathbb{D} \backslash \mathcal{S}$, then $N$ is also an invariant set under the flow $\Phi$ of $p(\mathcal{X})$, and the flow $\left(N,\left.\Phi\right|_{N}\right)$ is called a canonical region of the flow $(\mathbb{D}, \Phi)$.

Proposition 5. If the number of separatrices of the flow $(\mathbb{D}, \Phi)$ is finite, then every canonical region of the flow $(\mathbb{D}, \Phi)$ is parallel.

For a proof of this proposition see [16] or [11].

The separatrix configuration $\mathcal{S}_{c}$ of a flow $(\mathbb{D}, \Phi)$ is the union of all the separatrices $\mathcal{S}$ of the flow together with an orbit belonging to each canonical region. The separatrix configuration $\mathcal{S}_{c}$ of the flow $(\mathbb{D}, \Phi)$ is said to be topologically equivalent to the separatrix configuration $\mathcal{S}_{c}^{*}$ of the flow $\left(\mathbb{D}, \Phi^{*}\right)$ if there exists a homeomorphism from $\mathbb{D}$ to $\mathbb{D}$ which transforms orbits of $\mathcal{S}_{c}$ into orbits of $\mathcal{S}_{c}^{*}$ preserving or reversing the sense of all orbits.

Theorem 6 (Markus-Neumann-Peixoto). Let $(\mathbb{D}, \Phi)$ and $\left(\mathbb{D}, \Phi^{*}\right)$ be two compactified Poincaré flows with finitely many separatrices coming from two polynomial vector fields (2). Then they are topologically equivalent if and only if their separatrix configurations are topologically equivalent.

For a proof of this result we refer the reader to $[14,16,17]$.

It follows from the previous theorem that in order to classify the phase portraits in the Poincaré disc of a planar polynomial differential system having finitely many separatrices finite and infinite, it is enough to describe their separatrix configuration.

Finally, Bautin [1] showed that the Lotka-Volterra systems cannot have a limit cycle, therefore we only look for the other types of separatrices.

\section{Proof of Theorem 2}

We consider the family of quadratic Lotka-Volterra systems (4). First, we calculate its finite singularities and we find that if $a c \neq 0$ then there are two singular points $(0,0)$ and $(-c / a, 0)$. In the cases $a=0$ and $c \neq 0$, or $a \neq 0$ and $c=0$, we have only the singular point $(0,0)$; and if $a=0$ and $c=0$ there exist infinitely many singular 


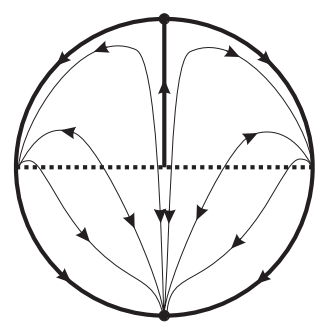

FigURE 1. Phase portrait of system (11) when $b \neq 0$. It has infinitely many separatrices.

points of the form $(x, 0)$ with $x \in \mathbb{R}$. Recall that $C \neq 0$ in order to guarantee the existence of a Darboux invariant.

As we are interested in classifying the phase portraits in the Poincaré disc of system (4), we introduce a rescaling of the variables and of the time to reduce the number of its parameters as much as possible, and hence the phase portraits obtained for the new system will be topologically equivalent to the corresponding phase portraits of system (4). Let

$$
x=\alpha X, \quad y=\beta Y \quad \text { and } \quad t=\gamma T
$$

where $\alpha, \beta$, and $\gamma$ are parameters.

Case 1: $a=c=0$. Then system (4) becomes

$$
\dot{x}=b x y, \quad \dot{y}=C y,
$$

whose singularities $(x, 0)$ are not isolated points. System $(11)$ written in the new variables $X$ and $Y$ is

$$
X^{\prime}=\gamma b \beta X Y, \quad Y^{\prime}=\gamma C Y,
$$

where' denotes the derivative with respect to the variable $T$. Taking $\gamma C=1$ and $\gamma b \beta=1$ it follows that $\gamma=1 / C$ and, moreover $\beta=C / b$ when $b \neq 0$.

If $b=0$, system (12) is $X^{\prime}=0, Y^{\prime}=Y$, since it is a linear differential system we do not consider it.

Let $b \neq 0$, then system $(12)$ is

$$
X^{\prime}=X Y, Y^{\prime}=Y .
$$

Therefore system (13) possesses infinitely many finite singularities, the points $(X, 0)$. Resolving this quadratic system we obtain solutions $Y=\ln |X|+k$ with $k \in \mathbb{R}$ and $X \neq 0$. The expression for $p(\mathcal{X})$ in the local chart $U_{1}$ is $u^{\prime}=-u^{2}+u v, v^{\prime}=-u v$ which possesses a unique singularity on $U_{1}$, the origin.

On $U_{2}$ the expression for $p(\mathcal{X})$ is $u^{\prime}=u-u v, v^{\prime}=-v^{2}$. So the origin is a singular point on $U_{2}$. By Theorem 2.19 of [6], it is a semi-hyperbolic saddle-node with stable separatrix tangent at $(0,0)$ to the positive $v$-axis. The two unstable separatrices are tangent to the $u$-axis in $(0,0)$. Therefore, by joining all the previous information we obtain the phase portrait of quadratic system (11) on the Poincaré disc when $b \neq 0$, which is topologically equivalent to the one shown in Figure 1.

In the figures we shall use the following notation. The singular points are denoted by dots, the separatrices which are non singular points are denoted by thicker lines, and an orbit inside a canonical region is represented by a thinner line. 
Case 2: $a \neq 0$ and $c=0$. Hence system (4) is

$$
\dot{x}=x(a x+b y), \quad \dot{y}=C y .
$$

System (14) written in the variables $X$ and $Y$, according to (10), becomes

$$
X^{\prime}=X(\gamma a \alpha X+\gamma b \beta Y), \quad Y^{\prime}=\gamma C Y,
$$

Taking $\gamma C=1, \gamma a \alpha=1$ and $\gamma b \beta=1$ it follows that $\gamma=1 / C$ and $\alpha=C / a$. Moreover $\beta=C / b$ when $b \neq 0$.

If $b=0$, system $(15)$ is

$$
X^{\prime}=X^{2}, \quad Y^{\prime}=Y .
$$

This system has only a finite singularity, the origin, which is a semi-hyperbolic saddlenode with stable separatrix tangent at $(0,0)$ to the negative $\mathrm{X}$-axis and two unstable separatrices tangent at $(0,0)$ to the $\mathrm{Y}$-axis. The expression for the Poincare compactification $p(\mathcal{X})$ in the local chart $U_{1}$ is $u^{\prime}=-u+u v, v^{\prime}=-v$. Therefore on $U_{1}$ the origin is the unique singular point, which is a hyperbolic stable node at infinity. Since on $U_{1}$ the degree of $p(\mathcal{X})$ is 2 , the diametrally opposite point is a hyperbolic unstable node in $V_{1}$.

The expression for $p(\mathcal{X})$ in the local chart $U_{2}$ is

$$
u^{\prime}=u^{2}-u v, \quad v^{\prime}=-v^{2} .
$$

So the origin of $U_{2}$ is a singular point linearly zero. Since system (17) is homogeneous we apply the classification due to Date [5], see the appendix. Using the definitions given at the appendix, we have that $D=0,\left[h^{\kappa \lambda}\right]=\left[\begin{array}{cc}0 & 0 \\ 0 & -1 / 9\end{array}\right] \neq 0$ and $H=-1 / 4 \neq$ 0 . So system (17) belongs to the type II(1). Moreover the value of the covariant $K_{2}$ is $27 / 8>0$. Therefore we obtain that system (17) is of Type II(1-1). In consequence the phase portrait in the Poincaré disc of system (14) if $b=0$ is topologically equivalent to the one showed in Figure 2 with $b=0$.

If $b \neq 0$ we have that system (15) is $X^{\prime}=X(X+Y), Y^{\prime}=Y$. This system possesses only a finite singularity, the origin which is a semi-hyperbolic saddle-node with stable separatrix tangent at $(0,0)$ to the negative $\mathrm{X}$-axis and unstable separatrices tangent to the $\mathrm{Y}$-axis at $(0,0)$. The expression for $p(\mathcal{X})$ in the local chart $U_{1}$ is $u^{\prime}=-u-u^{2}+u v$, $v^{\prime}=-v-u v$. Thus there are two infinite singularities on $U_{1},(0,0)$ and $(-1,0)$. The origin is a hyperbolic stable node. The singular point $(-1,0)$ is a hyperbolic saddlenode whose stable separatrix is tangent at $(-1,0)$ to the positive $v$-axis. The unstable separatrices are tangent at $(-1,0)$ to the $u$-axis.

The expression for $p(\mathcal{X})$ in the local chart $U_{2}$ is $u^{\prime}=u+u^{2}-u v, v^{\prime}=-v^{2}$. Therefore the origin is the unique singularity on $U_{2}$ which is a semi-hyperbolic saddlenode, its stable separatrix is tangent at origin to the positive $v$-axis and the unstable separatrices to the $u$-axis. So we get the phase portrait of system (14) if $b \neq 0$, which is topologically equivalent to the one shown in Figure 2 with $b \neq 0$.

Case 3: $a=0$ and $c \neq 0$. In this case system (4) is

$$
\dot{x}=x(b y+c), \quad \dot{y}=C y .
$$




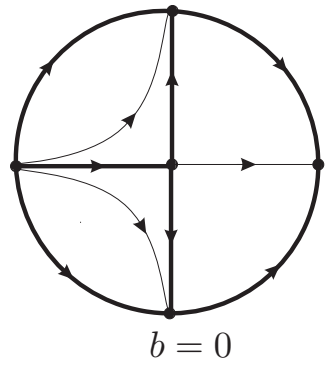

S: 12, CR: 3

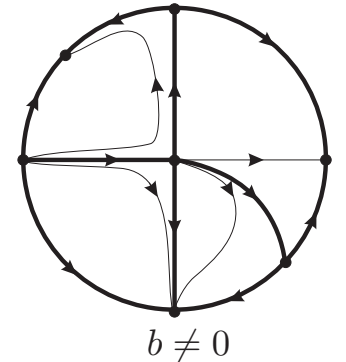

S: $17, \mathrm{CR}: 4$

FiguRE 2. Phase portraits of system (14). S is the number of all separatrices and $\mathrm{CR}$ is the number of all canonical regions.

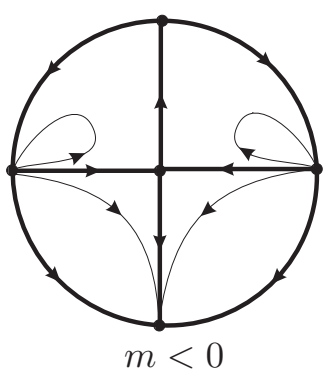

S: 13, CR: 4 .

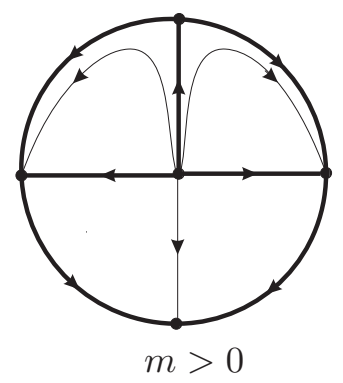

S: $12, \mathrm{CR}: 3$.

Figure 3. Phase portraits of system (18) with $m=c / C$ and $b \neq 0$.

Again from (10) we obtain that system (18) is transformed into $X^{\prime}=X(b \beta \gamma Y+$ $c \gamma), \quad Y^{\prime}=C \gamma Y$, and hence we obtain the two systems $X^{\prime}=\frac{c}{C} X, Y^{\prime}=Y$ if $b=0$, and $X^{\prime}=X(Y+c / C), Y^{\prime}=Y$ when $b \neq 0$.

We only consider the case $b \neq 0$ because for $b=0$ the system is linear, then the previous system is topologically equivalent to the system $X^{\prime}=X(Y+m), Y^{\prime}=Y$, respectively, with $m=c / C$ a nonzero constant. The only finite singular point is $(0,0)$ which is a hyperbolic unstable node if $m>0$, or a hyperbolic saddle if $m<0$. The expression for $p(\mathcal{X})$ in $U_{1}$ is $u^{\prime}=-u^{2}+(1-m) u v, v^{\prime}=-u v-m v^{2}$. This system is homogeneous and it possesses a unique singular point, the origin, which is linearly zero. We apply the appendix in order to study this singularity. Thus we obtain $D=0,\left[h^{\kappa \lambda}\right]=\left[\begin{array}{cc}-1 / 9 & 0 \\ 0 & 0\end{array}\right] \neq 0$ and $H=-1 / 4 \neq 0$. So the system belongs to the type $\mathrm{II}(1)$. Moreover $K_{2}=27 \mathrm{~m} / 8$, in consequence if $m>0$, the system is of the type $\mathrm{II}(1-1)$, and it is of type $\mathrm{II}(1-2)$ if $m<0$.

In $U_{2}$ the expression for $p(\mathcal{X})$ is $u^{\prime}=u+(m-1) u v, v^{\prime}=-v^{2}$. So $U_{2}$ contains only a singularity, the origin, which is a semi-hyperbolic saddle-node with stable separatrix tangent at the origin to the positive $v$-axis. The two unstable separatrices are tangent at $(0,0)$ to the $u$-axis. In Figure 3 are shown the phase portraits topologically equivalent to the ones of system (18) for $b \neq 0$.

Case 4: $a \neq 0$ and $c \neq 0$. Hence system (4) becomes

$$
\dot{x}=a x^{2}+b x y+c x, \quad \dot{y}=C y .
$$




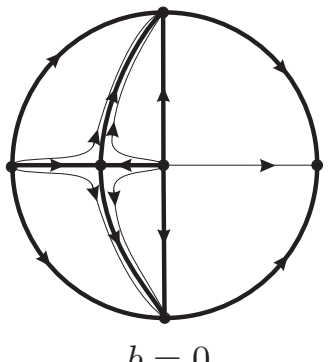

S: 16, CR: 5 .

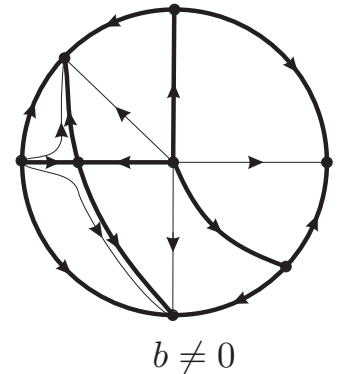

S: 20, CR: 5 .

Figure 4. Phase portraits of system (19).

From (10) we have that system (19) is topologically equivalent to one of the two systems $X^{\prime}=X^{2}+c / C X, Y^{\prime}=Y$ if $b=0$ or $X^{\prime}=X^{2}+X Y+c / C X, Y^{\prime}=Y$ if $b \neq 0$.

We analyze first the case $b=0$. Consider the system $X^{\prime}=X^{2}+m X, Y^{\prime}=Y$ with $m \neq 0$. Finite singularities are $(0,0)$ and $(-m, 0)$. The singular point $(0,0)$ is a hyperbolic unstable node if $m>0$ and a hyperbolic saddle if $m<0$. Also $(-m, 0)$ is a hyperbolic saddle if $m>0$ and a hyperbolic unstable node if $m<0$. The expression of $p(\mathcal{X})$ in $U_{1}$ is $u^{\prime}=-u+(1-m) u v, v^{\prime}=-v-m v^{2}$. There is a unique singularity on $U_{1}$, the origin, which is a hyperbolic stable node.

In local chart $U_{2}, p(\mathcal{X})$ is $u^{\prime}=u^{2}+(m-1) u v, v^{\prime}=-v^{2}$. So the origin is a singular point on $U_{2}$ linearly zero. Since this system is homogeneous we can use the appendix for its classification. Hence $D=-m^{2} / 27<0$ and so the system belongs to the type $\mathrm{I} \ominus$. Moreover since $K_{2}=27 / 8>0$, the system is of type $\mathrm{I} \ominus(1)$.

Joining all the above information we get the two phase portraits of the system (19) when $b=0$ corresponding to the cases $m<0$ or $m>0$. However, it is not difficult to verify that these two configurations are topologically equivalent. In Figure 4 with $b=0$ is presented a phase portrait topologically equivalent to the one of system (19) when $b=0$.

If $b \neq 0$, we consider the system $X^{\prime}=X^{2}+X Y+m X, Y^{\prime}=Y$ with $m \neq 0$. There are two finite singularities, $(0,0)$ and $(-m, 0)$. The origin is a hyperbolic unstable node if $m>0$, and it is a hyperbolic saddle if $m<0$. The singular point $(-m, 0)$ is a hyperbolic saddle if $m>0$ and it is a hyperbolic unstable node if $m<0$. The expression of $p(\mathcal{X})$ on $U_{1}$ is $u^{\prime}=-u-u^{2}+(1-m) u v, v^{\prime}=-v-u v-m v^{2}$. So the singular points on $U_{1}$ are $(0,0)$ and $(-1,0)$. The origin is a stable node and $(-1,0)$ is a semi-hyperbolic saddle-node, its stable separatrix is tangent to the $v$-axis at $(-1,0)$ and the unstable separatrices are tangent to the $u$-axis at $(-1,0)$.

In $U_{2}$ the expression for $p(\mathcal{X})$ is $u^{\prime}=u+u^{2}+(m-1) u v, v^{\prime}=-v^{2}$. Therefore the origin of $U_{2}$ is a singular point which is a semi-hyperbolic saddle-node with stable separatrix tangent at $(0,0)$ to the positive $v$-axis. The two unstable separatrices are tangent at $(0,0)$ to the $u$-axis. So we obtain the two phase portraits for $m<0$ and $m>0$. It is easy to see that these two configurations are topologically equivalent. Figure 4 with $b \neq 0$ shows a phase portrait topologically equivalent to the one of system (4) in this case. This completes the proof of Theorem 2. 


\section{Proof of Theorem 3}

We will find the phase portraits in the Poincaré disc of any quadratic Lotka-Volterra system (5). For doing this we introduce a rescaling of the variables and of the time in order to reduce the number of parameters of this system as much as possible. Obtaining the phase portraits for each of the new systems we shall get the phase portraits of the corresponding systems (5). Let $x=\alpha X, y=\beta Y$ and $t=\gamma T$, where $\alpha, \beta$ and $\gamma$ are parameters. In the new variables $X, Y$ and $T$ system (5) becomes

$$
X^{\prime}=X\left(-\frac{l_{2}}{l_{1}} A \alpha \gamma X-\frac{l_{2}}{l_{1}} B \beta \gamma Y+c \gamma\right), \quad Y^{\prime}=Y(A \alpha \gamma X+B \beta \gamma Y+\gamma C),
$$

here ' denotes the derivative with respect to the variable $T$. We recall that $l_{1} l_{2} \neq 0$ and $c^{2}+C^{2} \neq 0$.

We consider the following cases. Note that $A=B=0$ provides a linear differential system, and consequently it is not considered here.

Case 1: $A \neq 0, B=0$. Then system (20) is

$$
X^{\prime}=X\left(-\frac{l_{2}}{l_{1}} A \alpha \gamma X+c \gamma\right), \quad Y^{\prime}=Y(A \alpha \gamma X+C \gamma) .
$$

In order to study system (20) we consider three subcases.

Subcase 1.1: $C=0, c \neq 0$. Therefore system (21) is $X^{\prime}=X\left(-l_{2} A \alpha \gamma X / l_{1}+c \gamma\right)$, $Y^{\prime}=A \alpha \gamma X Y$. Taking $\gamma=1 / c$ and $\alpha=c / A$, the previous system is converted into

$$
X^{\prime}=X+m X^{2}, \quad Y^{\prime}=X Y,
$$

where $m=l_{2} / l_{1}$. System (22) has infinitely many finite singularities, the points $(0, Y)$. Doing $d s=X d T$, system $(22)$ becomes

$$
d X / d s=m X+1, \quad d Y / d s=Y
$$

with $m \neq 0$. System (23) has only a unique singularity, $(-1 / m, 0)$. It is a hyperbolic saddle when $m<0$, and it is a hyperbolic unstable node if $m>0$. The expression of $p(\mathcal{X})$ in the local chart $U_{1}$ is $u^{\prime}=(1-m) u-u v, v^{\prime}=-m v-v^{2}$. Then only the origin is a singular point if $m \neq 1$. However, there are infinitely many singularities if $m=1$, the points $(u, 0)$. If $m \neq 1,(0,0)$ is a hyperbolic unstable node if $m<0$, it is a hyperbolic saddle if $0<m<1$, and a hyperbolic stable node when $m>1$.

In $U_{2}$ the expression of $p(\mathcal{X})$ is $u^{\prime}=(m-1) u+v, v^{\prime}=-v$. So the origin is a singular point. If $m>1$, it is a hyperbolic saddle, and a hyperbolic stable node if $m<1$. Since the rescaling $d s=X d T$ changes the orientation of orbits where $X$ is negative, we obtain that the phase portraits of system (22) are topologically equivalent to the ones shown in Figure 5.

Subcase 1.2: $C \neq 0, c=0$. So system $(21)$ is $X^{\prime}=-l_{2} A \alpha \gamma / l_{1} X^{2}, Y^{\prime}=Y(A \alpha \gamma X+$ $\gamma C)$. Taking $\gamma=1 / C$ and $\alpha=C / A$ we obtain

$$
X^{\prime}=m X^{2}, \quad Y^{\prime}=X Y+Y,
$$

with $m=-l_{2} / l_{1} \neq 0$. The origin is the unique finite singularity of system (24), which is a semi-hyperbolic saddle-node. If $m>0$, its stable separatrix is tangent at $(0,0)$ to the negative $X$-axis, and to the positive $X$-axis when $m<0$. The expression of 

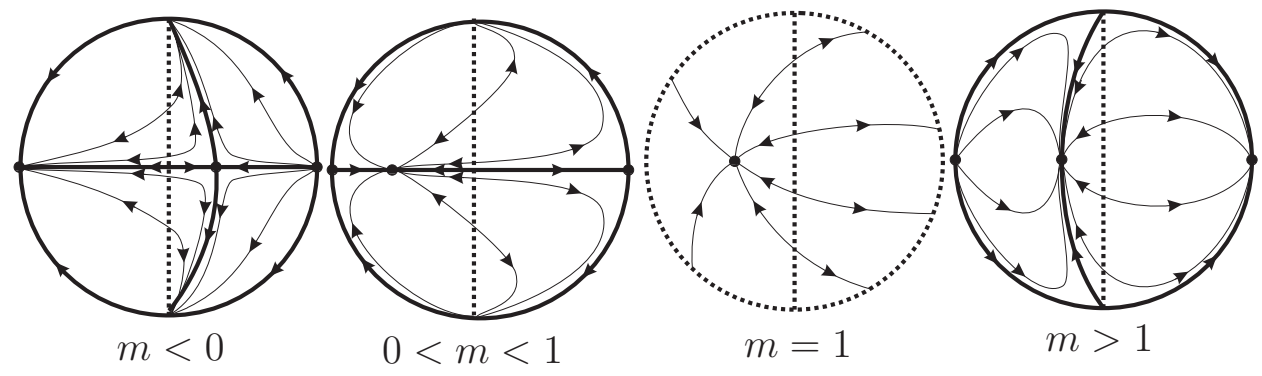

FIGURE 5. Phase portraits of system (22). They have infinitely many separatrices.

$p(\mathcal{X})$ in the local chart $U_{1}$ is $u^{\prime}=(1-m) u+u v, v^{\prime}=-m v$. If $m \neq 1$, on $U_{1}$ there is only one singular point, the origin; if $m<0,(0,0)$ is a hyperbolic unstable node, or it is a hyperbolic saddle when $0<m<1$, or a hyperbolic stable node if $m>1$. If $m=1$, the system becomes $u^{\prime}=u v, v^{\prime}=-v$ which possesses infinitely many singularities, the points $(u, 0)$.

The expression of $p(\mathcal{X})$ in $U_{2}$ is $u^{\prime}=-u v+(m-1) u^{2}, v^{\prime}=-u v-v^{2}$. Therefore, if $m \neq 1$ we have that $(0,0)$ is a linearly zero singular point and the system is homogeneous. Applying the appendix we obtain that $D=0,\left[h^{\kappa \lambda}\right]=\left[\begin{array}{cc}0 & 0 \\ 0 & -m^{2} / 9\end{array}\right] \neq 0$ and $H=-m^{2} / 4 \neq 0$. In consequence the system belongs to the type $\mathrm{II}(1)$. As $K_{2}=27 m(m-1) / 8$, then the system is of the type II(1-1) if $K_{2}>0$, i.e. if $m<0$ or $m>1$. If $0<m<1$ then $K_{2}<0$ and so the system is of type II(1-2). If $m=1$, then the system belongs to the type II(1-3).

Therefore we obtain the phase portraits of system (24), which are topologically equivalent to the phase portrait for $b=0$ of Figure 2 when $m<0$ or $m>1$, and the others are topologically equivalent to the ones represented in Figure 6.

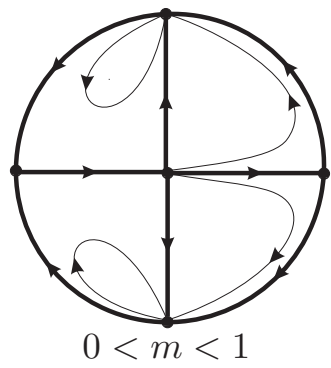

S: 13, CR: 4 .

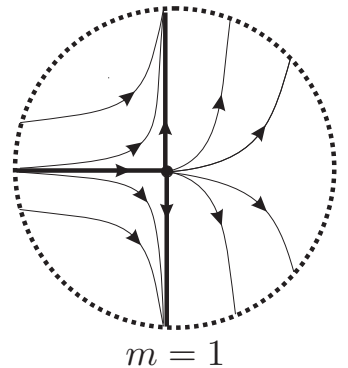

Infinitely many separatrices.

FIGURE 6. Phase portraits of system (24).

Subcase 1.3: $C \neq 0, c \neq 0$. Hence taking $\gamma=1 / C$ and $\alpha=C / A$ in system (21) we obtain

$$
X^{\prime}=m_{1} X^{2}+m_{2} X, \quad Y^{\prime}=Y+X Y,
$$

where $m_{1}=-l_{2} / l_{1} \neq 0$ and $m_{2}=c / C \neq 0$. So if $m_{1} \neq m_{2}$, system (25) has only two finite singular points, the points $(0,0)$ and $\left(-m_{2} / m_{1}, 0\right)$, and it has infinitely many singularities when $m_{1}=m_{2}$, all the points of the straight line $X=-1$ and $(0,0)$. 
The origin is a hyperbolic saddle if $m_{2}<0$, and it is a hyperbolic unstable node if $m_{2}>0$. If $m_{1} \neq m_{2}$, the Jacobian matrix at $\left(-m_{2} / m_{1}, 0\right)$ is $\left(\begin{array}{cc}-m_{2} & 0 \\ 0 & 1-m_{2} / m_{1}\end{array}\right)$. The point $\left(-m_{2} / m_{1}, 0\right)$ is a hyperbolic singularity. It is an unstable node if $m_{1}<$ $m_{2}<0$, or if $m_{1}>0$ and $m_{2}<0$. It is a saddle when $m_{1}<0$ and $m_{2}>0$, or if $m_{2}<m_{1}<0$, or if $m_{1}>m_{2}>0$. Moreover if $0<m_{1}<m_{2}$, it is a stable node.

Now we study system $(25)$ at infinity. The expression of $p(\mathcal{X})$ in the local chart $U_{1}$ is $u^{\prime}=\left(1-m_{1}\right) u+\left(1-m_{2}\right) u v, v^{\prime}=-m_{1} v-m_{2} v^{2}$. Therefore when $m_{1} \neq 1$, there is a unique singular point at $U_{1}$ the origin, which is hyperbolic. If $m_{1}<0,(0,0)$ is an unstable node. If $0<m_{1}<1$, the origin is a saddle; and $(0,0)$ is a hyperbolic stable node when $m_{1}>1$. However, there are infinitely many singularities at $U_{1}$ when $m_{1}=1$, the points $(u, 0)$.

On $U_{2}$ the Poincaré compactification $p(\mathcal{X})$ is

$$
u^{\prime}=\left(m_{1}-1\right) u^{2}+\left(m_{2}-1\right) u v, \quad v^{\prime}=-u v-v^{2} .
$$

So $(0,0)$ is a linearly zero singularity. Since system $(26)$ is homogeneous we use the appendix for its classification. Then, since $D=-m_{1}^{2} m_{2}^{2} / 27<0$ we get that system (26) belongs to the type I $\ominus$. Computing the values of the covariants $K_{2}=$ $27\left(m_{1}-1\right)\left(m_{1}-m_{2}\right) / 8$ and $K_{3}=27\left(m_{2}-1\right)\left(m_{1}-m_{2}+m_{1} m_{2}\right) / 8$, we obtain the following classification for system $(26)$.

\begin{tabular}{|c|c|c|c|}
\hline Condition & Sign of $K_{2}$ and $K_{3}$ & Type & Phase portrait \\
\hline$m_{1}<0, m_{1}<m_{2} ; m_{1}>1, m_{1}>m_{2}$ & \multirow[t]{2}{*}{$K_{2}>0$} & \multirow[t]{2}{*}{$\mathrm{I} \ominus(1)$} & $b=0$ of Fig. 4, \\
\hline $0<m_{1}<1, m_{1}<m_{2}$ & & & (1) of Fig. 7 \\
\hline$m_{1}>1, m_{1}<m_{2}, m_{2}>1$ & $K_{2}<0, K_{3}>0$ & $\mathrm{I} \ominus(2)$ & (2) of Fig. 7 \\
\hline$m_{1}<0, m_{1}>m_{2}, m_{2}<0$ & \multirow[t]{2}{*}{$K_{2}<0, K_{3}<0$} & \multirow[t]{2}{*}{$\mathrm{I} \ominus(3)$} & (3) of Fig. 7 \\
\hline $0<m_{1}<1, m_{1}>m_{2}, m_{2}<1$ & & & (4) of Fig. 7 \\
\hline$m_{1}=1, m_{2}>1$ & \multirow[t]{2}{*}{$K_{2}=0, K_{3}>0$} & \multirow[t]{2}{*}{$\mathrm{I} \ominus(4)$} & (6) of Fig.7, \\
\hline$m_{1}=m_{2}, m_{2}>1$ & & & $m>1$ of Fig. 5 \\
\hline$m_{1}=1, m_{2}<1$ & \multirow[t]{2}{*}{$K_{2}=0, K_{3}<0$} & \multirow[t]{2}{*}{$\mathrm{I} \ominus(5)$} & (5) of Fig. 7, \\
\hline$m_{1}=m_{2}, m_{2}<1$ & & & $m<0,0<m<1$ of Fig. 5 \\
\hline$m_{1}=1, m_{2}=1$ & $K_{2}=0=K_{3}$ & $\mathrm{I} \ominus(6)$ & $m=1$ of Fig. 5 \\
\hline
\end{tabular}

In consequence we obtain the phase portraits of system (25), which are topologically equivalent to the phase portrait for $b=0$ of Figure 4 when $m_{1}<0$ and $m_{1}<m_{2}$ or when $m_{1}>1$ and $m_{1}>m_{2}$. Or to the phase portrait for $m<0$ of Figure 5 when $m_{1}=m_{2}<0$, to the phase portrait for $0<m<1$ of Figure 5 when $0<m_{1}=m_{2}<1$, to the phase portrait for $m=1$ of Figure 5 when $m_{1}=m_{2}=1$, and to the phase portrait for $m>1$ of Figure 5 when $m_{1}=m_{2}>1$ and the others are topologically equivalent to the ones of Figure 7.

Case 2: $A=0, B \neq 0$. So system (20) becomes

$$
X^{\prime}=X\left(-\frac{l_{2}}{l_{1}} B \beta \gamma Y+c \gamma\right), \quad Y^{\prime}=Y(B \beta \gamma Y+\gamma C) .
$$

Subcase 2.1: $C=0, c \neq 0$. System (27) for these values becomes $X^{\prime}=X\left(-l_{2} / l_{1} B \beta \gamma Y+\right.$ $c \gamma), Y^{\prime}=B \beta \gamma Y^{2}$. Taking $\gamma=1 / c$ and $\beta=-l_{1} /\left(B \gamma l_{2}\right)$ we obtain

$$
X^{\prime}=X Y+X, \quad Y^{\prime}=-l_{1} / l_{2} Y^{2} .
$$




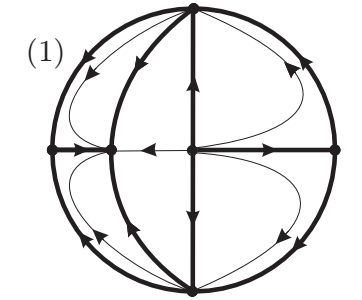

$0<m_{1}<1, m_{2}>0, m_{1}<m_{2}$, S: 16, CR: 5 .

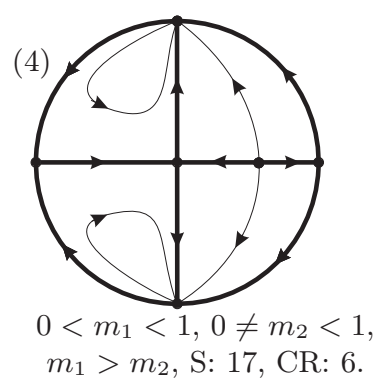

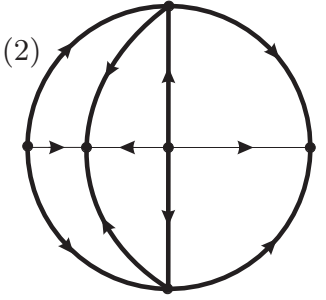

$1<m_{1}<m_{2}$

S: 14, CR: 3 .

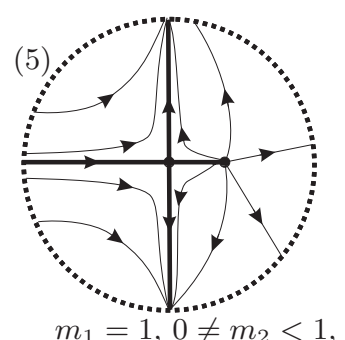

infinitely many separatrices.

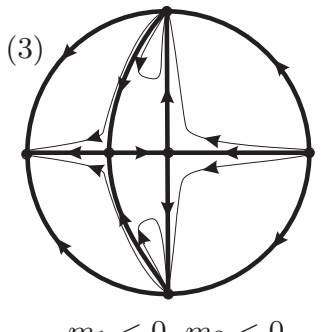

$m_{1}<0, m_{2}<0$, $m_{1}>m_{2}$, S: 17, CR: 6 .

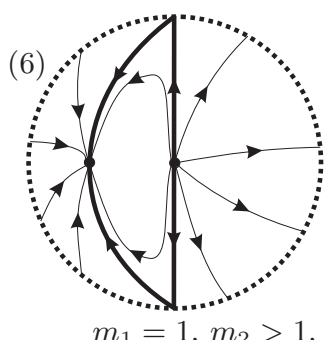

infinitely many separatrices.

FiguRe 7. Phase portraits of system (25).

Interchanging the variables $X$ and $Y$ we get the system (24) now with $m=-l_{1} / l_{2}$. Then the phase portraits of system (28) are the phase portrait for $b=0$ of Figure 2 when $m>-1$, and the phase portraits of Figure 6 but from the left to the right they correspond to the values $m<-1$ and $m=-1$.

Subcase 2.2: $C \neq 0, c=0$. So system (27) is $X^{\prime}=-l_{2} / l_{1} B \beta \gamma X Y, Y^{\prime}=\gamma C Y+$ $B \beta \gamma Y^{2}$. Taking $\gamma=1 / C$ and $\beta=C / B$ it becomes

$$
X^{\prime}=-m X Y, \quad Y^{\prime}=Y^{2}+1,
$$

where $m=l_{2} / l_{1} \neq 0$. System (29) does not possess real finite singularities.

On the local chart $U_{1}$ the expression of $p(\mathcal{X})$ is $u^{\prime}=(m+1) u^{2}+v^{2}, v^{\prime}=m u v$. So if $m \neq-1$ the origin is the unique singular point and it is linearly zero. Since this system is homogeneous, we use the appendix for its classification. Thus we obtain $D=4 / 27>0$, and so the system is of type $\mathrm{I} \oplus$. Moreover since $K_{2}=27 m^{2}(m+1) / 8$ we have that if $m>-1$, then $K_{2}>0$ and so the system is of type $\mathrm{I} \oplus(1)$. In the case $m<-1$ we obtain $K_{2}<0$, therefore the system is of type I $\oplus(2)$. If $m=-1$, then $K_{2}=0$, and the system is of type $\mathrm{I} \oplus(3)$.

The expression of $p(\mathcal{X})$ in $U_{2}$ is $u^{\prime}=-(m+1) u-u v^{2}, v^{\prime}=-v-v^{3}$. So $(0,0)$ is a singular point which is a hyperbolic saddle if $m<-1$ and it is a hyperbolic stable node when $m>-1$. In consequence, we obtain the phase portraits of system (29), which are topologically equivalent to the ones shown in Figure 8.

Subcase 2.3: $C \neq 0, c \neq 0$. So from system (27) and taking $\gamma=1 / c$ and $\beta=c / B$ we obtain

$$
X^{\prime}=X-m_{1} X Y, \quad Y^{\prime}=m_{2} Y+Y^{2},
$$




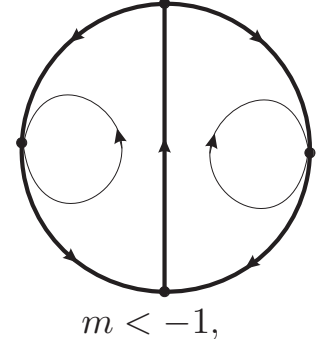

S: 9, CR: 2 .

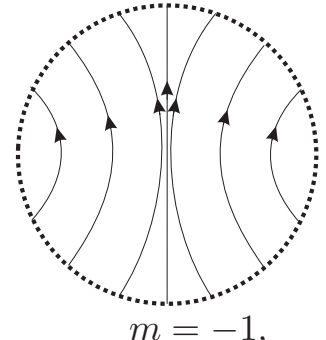

infinitely many separatrices.

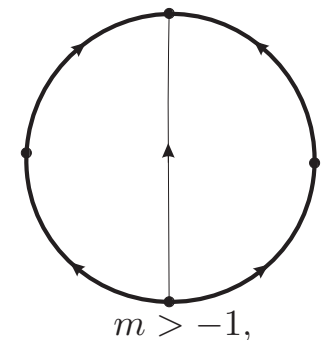

S: 8, CR: 1 .

FiguRE 8. Phase portraits of system (29).

where $m_{1}=l_{2} / l_{1} \neq 0$ and $m_{2}=C / c \neq 0$. The finite singularities of system (30) are the hyperbolic singular points $(0,0)$ and $\left(0,-m_{2}\right)$. The point $(0,0)$ is a saddle if $m_{2}<0$, and it is an unstable node when $m_{2}>0$.

Since $D \mathcal{X}\left(0,-m_{2}\right)=\left[\begin{array}{cc}1+m_{1} m_{2} & 0 \\ 0 & -m_{2}\end{array}\right]$, first we study the case $1+m_{1} m_{2}=0$. Then $m_{1}=-1 / m_{2}$ and system (30) becomes

$$
X^{\prime}=X\left(m_{2}+Y\right) / m_{2}, \quad Y^{\prime}=Y\left(m_{2}+Y\right) .
$$

We analyze the reduced system $X^{\prime}=X / m_{2}, Y^{\prime}=Y$ whose unique singular point is the origin. If $m_{2}>0$, then $(0,0)$ is a hyperbolic unstable node, and if $m_{2}<0$, it is a hyperbolic saddle. On the local chart $U_{1}$, the expression of $p(\mathcal{X})$ is $u^{\prime}=\left(1-1 / m_{2}\right) u$, $v^{\prime}=-v / m_{2}$ then $(0,0)$ is the unique singularity on $U_{1}$, which is a hyperbolic unstable node if $m_{2}<0$; or it is a hyperbolic stable node if $0<m_{2}<1$, or a hyperbolic saddle when $m_{2}>1$.

The expression of $p(\mathcal{X})$ on $U_{2}$ is $u^{\prime}=\left(1 / m_{2}-1\right) u, v^{\prime}=-v$. Therefore $(0,0)$ is a singular point, and it is hyperbolic. Moreover $(0,0)$ is a stable node if $m_{2}<0$ or $m_{2}>1$, and it is a saddle if $0<m_{2}<1$. In consequence we obtain the phase portraits of $X^{\prime}=X / m_{2}, Y^{\prime}=Y$. And so changing the orientation of orbits when $Y<-m_{2}$ we get the phase portraits of system (31).

If $1+m_{1} m_{2} \neq 0$, then we obtain that $\left(0,-m_{2}\right)$ is a stable node if $m_{2}>0$ and $m_{1}<-1 / m_{2}$, or, it is a saddle if $m_{2}>0$ and $m_{1}>-1 / m_{2}$, or $m_{2}<0$ and $m_{1}>-1 / m_{2}$, or an unstable node when $m_{2}<0$ and $m_{1}<-1 / m_{2}$.

On the local chart $U_{1}$, the expression of $p(\mathcal{X})$ is

$$
u^{\prime}=\left(m_{2}-1\right) u v+\left(m_{1}+1\right) u^{2}, \quad v^{\prime}=m_{1} u v-v^{2},
$$

which is a homogeneous system. So we can apply the classification of Date to system (32) (see appendix). Realizing the calculations we obtain $D=-m_{2}^{2} / 27<0$, thus system (32) belongs to the type I $\ominus$. Since $K_{2}=27\left(m_{1}+1\right)\left(m_{1} m_{2}+1\right) / 8$ and 
$K_{3}=-27 m_{1}\left(m_{2}-1\right)\left(1+m_{2}+m_{1} m_{2}\right) / 8$ the classification is given in the next table.

\begin{tabular}{|c|c|c|c|}
\hline Condition & $\begin{array}{l}\text { Sign of } K_{2} \\
\text { and } K_{3}\end{array}$ & Type & P.portrait \\
\hline$m_{1}<-1, m_{2}>0, m_{1}<-1 / m_{2}$ & \multirow{3}{*}{$K_{2}>0$} & \multirow{3}{*}{$\mathrm{I} \ominus(1)$} & (1) of Fig. 7 \\
\hline$m_{1}>-1, m_{2}>0, m_{1}>-1 / m_{2}$ & & & \multirow{2}{*}{$b=0$ of Fig. 4} \\
\hline$m_{1}>-1, m_{2}<0, m_{1}<-1 / m_{2}$ & & & \\
\hline$m_{1}<-1,0<m_{2}<1, m_{1}>-1 / m_{2}$ & \multirow[t]{2}{*}{$K_{2}<0, K_{3}<0$} & \multirow[t]{2}{*}{$\mathrm{I} \ominus(3)$} & \multirow[t]{2}{*}{ (4) of Fig. 7} \\
\hline$m_{1}<-1, m_{2}<0, m_{1}<-1 / m_{2}$ & & & \\
\hline$-1<m_{1}<0, m_{2}>1, m_{1}<-1 / m_{2}$ & $K_{2}<0, K_{3}>0$ & $\mathrm{I} \ominus(2)$ & (2) of Fig. 7 \\
\hline$m_{1}>0, m_{2}<0, m_{1}>-1 / m_{2}$ & $K_{2}<0, K_{3}<0$ & $\mathrm{I} \ominus(3)$ & (3) of Fig. 7 \\
\hline$m_{1}=-1, m_{2}>1$ & \multirow[t]{2}{*}{$K_{2}=0, K_{3}>0$} & \multirow[t]{2}{*}{$\mathrm{I} \ominus(4)$} & (6) of Fig. 7 \\
\hline$m_{1}=-1 / m_{2}, m_{2}>1$ & & & $0<m<1$ of Fig. 5 \\
\hline$m_{1}=-1, m_{2}<1$ & \multirow[t]{2}{*}{$K_{2}=0, K_{3}<0$} & \multirow[t]{2}{*}{$\mathrm{I} \ominus(5)$} & (5) of Fig. 7 \\
\hline$m_{1}=-1 / m_{2}, m_{2}<1$ & & & $0<m<1$ of Fig. 5 \\
\hline$m_{1}=-1, m_{2}=1$ & $K_{2}=0=K_{3}$ & $\mathrm{I} \ominus(6)$ & $m=1$ of Fig. 5 \\
\hline
\end{tabular}

The expression for $p(\mathcal{X})$ in the local chart $U_{2}$ is $u^{\prime}=\left(1-m_{2}\right) u v-\left(m_{1}+1\right) u$, $v^{\prime}=-v-m_{2} v^{2}$. Therefore $(0,0)$ is a singular point, which is a hyperbolic saddle if $m_{1}<-1$, and it is a hyperbolic stable node if $m_{1}>-1$. If $m_{1}=-1$ then system becomes $u^{\prime}=\left(1-m_{2}\right) u v, v^{\prime}=-v-m_{2} v^{2}$, which possesses infinitely many singularities, all the points $(u, 0)$. Consequently, we get the phase portraits in the Poincaré disc of system (30) for each one of cases that occur and they are topologically equivalent to the ones described in the previous table.

Case 3: $A B \neq 0$.

Subcase 3.1: $C=0, c \neq 0$. Hence system (20) is $X^{\prime}=X\left(-l_{2} / l_{1} A \alpha \gamma X-l_{2} / l_{1} B \beta \gamma Y+\right.$ $c \gamma), Y^{\prime}=Y(A \alpha \gamma X+B \beta \gamma Y)$. Taking $\gamma=1 / c, \alpha=c / A$ and $\beta=c / B$ we obtain

$$
X^{\prime}=X(1-m X-m Y), \quad Y^{\prime}=Y(X+Y),
$$

with $m=-l_{2} / l_{1} \neq 0$. The finite singularities of system (33) are the points $(0,0)$ and $(1 / m, 0)$. If $m>0$, then $(1 / m, 0)$ is a hyperbolic saddle, and it is a hyperbolic stable node when $m<0$. The origin is a semi-hyperbolic saddle-node with stable separatrix tangent to the negative $Y$-axis.

The expression of the Poincaré compactification $p(\mathcal{X})$ on the local chart $U_{1}$ is

$$
u^{\prime}=(m+1) u+(m+1) u^{2}-u v, \quad v^{\prime}=m v+m u v-v^{2} .
$$

So if $m \neq-1$, there are two singularities on $U_{1}$, the points $(0,0)$ and $(-1,0)$, which are hyperbolic. Moreover $(0,0)$ is a stable node if $m<-1$, it is a saddle when $-1<m<0$, and an unstable node if $m>0$. The point $(-1,0)$ is a semi-hyperbolic saddle-node.

If $m=-1$, then system (34) is $u^{\prime}=-u v, v^{\prime}=-v-u v-v^{2}$ which possesses infinitely many singularities $(u, 0)$ for all $u$.

On the local chart $U_{2}, p(\mathcal{X})$ is $u^{\prime}=-(m+1) u-(m+1) u^{2}+u v, v^{\prime}=-v-u v$. Then $(0,0)$ is a hyperbolic singular point. If $m<-1$, then $(0,0)$ is a saddle, and it is a stable node if $m>-1$. Therefore we obtain the phase portraits of system (33) which are topologically equivalent to the ones depicted in Figure 9. 


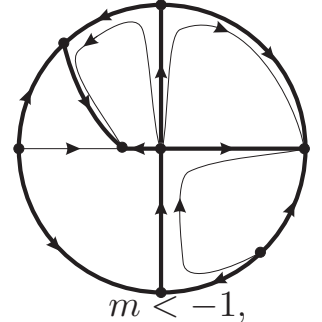

S: 19, CR: 4 .

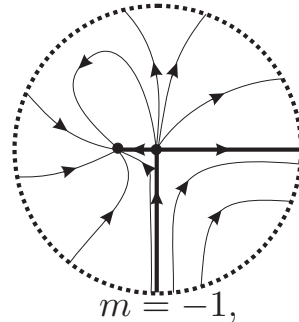

infinitely many separatrices.

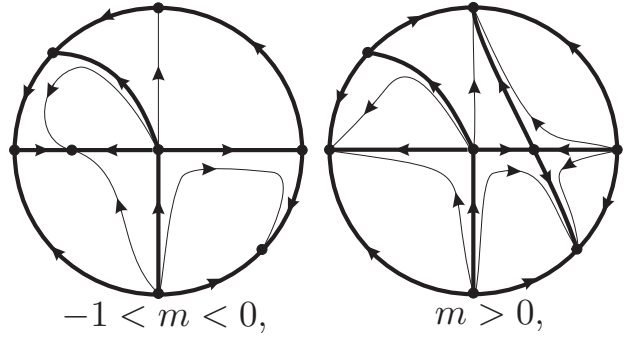

S: 21, CR: 6 .

FiguRE 9. Phase portraits of system (33).

Subcase 3.2: $C \neq 0, c=0$. Hence system (20) is $X^{\prime}=X\left(-l_{2} / l_{1} A \alpha \gamma X-l_{2} / l_{1} B \beta \gamma Y\right)$, $Y^{\prime}=Y(A \alpha \gamma X+B \beta \gamma Y+\gamma C)$. Let $\gamma=\frac{1}{C}, \alpha=-\frac{l_{1} C}{l_{2} A}$ and $\beta=-\frac{l_{1} C}{l_{2} B}$, then

$$
X^{\prime}=X(X+Y), \quad Y^{\prime}=Y(1-m X-m Y),
$$

where $m=l_{1} / l_{2} \neq 0$. So system (35) by interchanging the variables $X$ and $Y$ coincides with system (33).

Subcase 3.3: $C \neq 0, c \neq 0$. So taking $\gamma=1 / c, \alpha=c / A$ and $\beta=c / B$, system (20) is transformed into

$$
X^{\prime}=X-m_{1} X Y-m_{1} X^{2}, \quad Y^{\prime}=m_{2} Y+X Y+Y^{2},
$$

where $m_{1}=l_{2} / l_{1} \neq 0$ and $m_{2}=C / c \neq 0$. The finite singular points are $(0,0)$, $\left(1 / m_{1}, 0\right)$ and $\left(0,-m_{2}\right)$. The origin is a hyperbolic saddle if $m_{2}<0$, and it is a hyperbolic unstable node if $m_{2}>0$. We know that $m_{2} \neq-1 / m_{1}$, because otherwise, according to Theorem 1, system (36) would not possess a Darboux invariant. Thus the point $\left(1 / m_{1}, 0\right)$ is a hyperbolic singular point. It is a stable node if $m_{2}<-1 / m_{1}$, and it is a saddle if $m_{2}>-1 / m_{1}$. Also $\left(0,-m_{2}\right)$ is a hyperbolic singularity. It is an unstable node if $m_{2}<0$ and $m_{1}<-1 / m_{2}$; or it is a saddle when $m_{2}<0$ and $m_{1}>-1 / m_{2}$. If $m_{2}>0$ and $m_{1}<-1 / m_{2}$, then $\left(0,-m_{2}\right)$ is a stable node, and a saddle when $m_{2}>0$ and $m_{1}>-1 / m_{2}$.

The expression of $p(\mathcal{X})$ on $U_{1}$ is

$$
u^{\prime}=\left(1+m_{1}\right) u+\left(m_{2}-1\right) u v+\left(1+m_{1}\right) u^{2}, \quad v^{\prime}=m_{1} v+m_{1} u v-v^{2} .
$$

The singularities on $U_{1}$ are $(0,0)$ and $(-1,0)$ if $m_{1} \neq-1$. In this case, the origin is a hyperbolic singular point, it is a stable node if $m_{1}<-1$, or a saddle when $-1<m_{1}<0$, or an unstable node if $m_{1}>0$. The point $(-1,0)$ is a semi-hyperbolic saddle-node.

If $m_{1}=-1$, then system (37) becomes $u^{\prime}=\left(m_{2}-1\right) u v, v^{\prime}=-v-u v-v^{2}$ and so there are infinitely many singularities on $U_{1}$, all the points $(u, 0)$. Thus we obtain the phase portraits of system (36) when $m_{1}=-1$, which are topologically equivalent to the one depicted in Figure $10(5)$. Note that $m_{1}=-1$ and $m_{2}=1$ does not occur because in this case $m_{2}=-1 / m_{1}$.

The expression of $p(\mathcal{X})$ on $U_{2}$ is $u^{\prime}=-\left(1+m_{1}\right) u+\left(1-m_{2}\right) u v-\left(1+m_{1}\right) u^{2}$, $v^{\prime}=-v-u v-m_{2} v^{2}$. Then $(0,0)$ is a hyperbolic singularity, which is a saddle if $m_{1}<-1$, and it is a stable node if $m_{1}>-1$. Therefore we obtain the phase portraits 
of system (36) of the remaining cases, these are topologically equivalent to the ones represented in Figure 10. This completes the proof of Theorem 3.

(1)

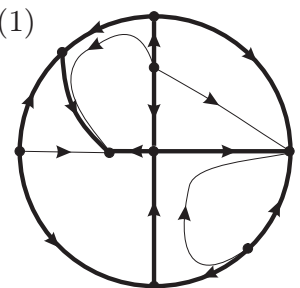

$m_{2}<0,-1 \neq m_{1}<0$ or $m_{2}>0, m_{1}<-1$, S: 21, CR: 4
$(2)$

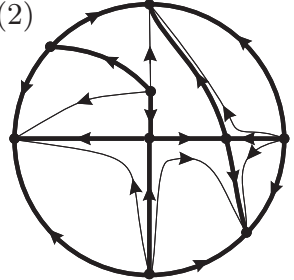

$m_{2}<0, m_{1}>0$, S: 23, CR: 6 .

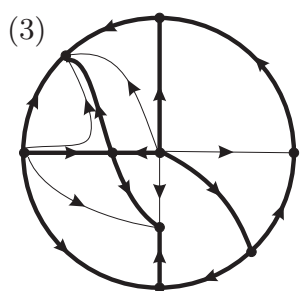

$m_{1}<-1, m_{1}>-1 / m_{2}$,

$0<m_{2}<1$, S: 22 , CR: 5 .
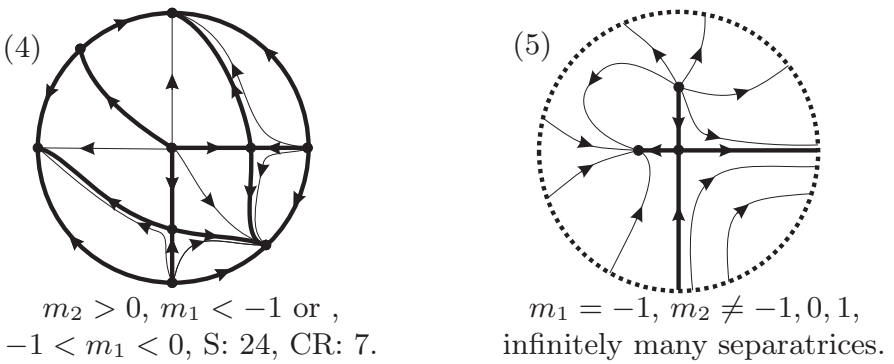

FIGURE 10. Phase portraits of system (36).

\section{APPENDIX: ON THE CLASSIFICATION OF TWO-DIMENSIONAL REAL HOMOGENEOUS QUADRATIC DIFFERENTIAL SYSTEMS}

We present some results on the classification of the phase portraits of two-dimensional real homogeneous quadratic differential systems due to Date [5].

A two-dimensional real homogeneous quadratic differential system is defined by

$$
\dot{x}=P_{11}^{1} x^{2}+2 P_{12}^{1} x y+P_{22}^{1} y^{2}, \quad \dot{y}=P_{11}^{2} x^{2}+2 P_{12}^{2} x y+P_{22}^{2} y^{2} .
$$

We first decompose $P_{\lambda \mu}^{k}$ into the "tensor part" $Q_{\lambda \mu}^{\kappa}$ and the "vector part" $p_{\lambda}$ as

$$
p_{\lambda}=P_{\lambda 1}^{1}+P_{\lambda 2}^{2}, \quad Q_{\lambda \mu}^{k}=P_{\lambda \mu}^{k}-\frac{1}{3}\left(\delta_{\lambda}^{k} p_{\mu}+\delta_{\mu}^{k} p_{\lambda}\right)
$$

where $\delta_{\lambda}^{\kappa}$ is the Kronecker delta and $k, \lambda, \mu=1,2$. Moreover $Q_{\lambda \mu}^{\kappa}=Q_{\mu \lambda}^{\kappa}$ and $Q_{\lambda 1}^{1}+$ $Q_{\lambda 2}^{2}=0$. 
Theorem 7. A two-dimensional real homogeneous quadratic differential system is affine-equivalent to one and only one of the following ten canonical forms

\begin{tabular}{|c|c|}
\hline type $\mathrm{I} \ominus$ & $\begin{array}{l}\dot{x}=-2 x y+2 x\left(\hat{p}_{1} x+\hat{p}_{2} y\right) / 3 \\
\dot{y}=-x^{2}+y^{2}+2 y\left(\hat{p}_{1} x+\hat{p}_{2} y\right) / 3\end{array}$ \\
\hline type $\mathrm{I} \oplus$ & $\begin{array}{l}\dot{x}=-2 x y+2 x\left(\hat{p}_{1} x+\hat{p}_{2} y\right) / 3, \\
\dot{y}=x^{2}+y^{2}+2 y\left(\hat{p}_{1} x+\hat{p}_{2} y\right) / 3\end{array}$ \\
\hline type $\mathrm{II}(1)$ & $\begin{array}{l}\dot{x}=\left(2 \hat{p}_{1} / 3-1\right) x^{2}+x y \\
\dot{y}=2\left(\hat{p}_{1}+3\right) x y / 3+y^{2}\end{array}$ \\
\hline type $\mathrm{II}(2)$ & $\begin{aligned} \dot{x} & =\left(2 \hat{p}_{1} / 3-1\right) x^{2} \\
\dot{y} & =2\left(\hat{p}_{1}+3\right) x y / 3\end{aligned}$ \\
\hline type $\operatorname{III}(1)$ & $\dot{x}=x y, \quad \dot{y}=x^{2}+y^{2}$ \\
\hline type $\operatorname{III}(2)$ & $\dot{x}=-x y, \quad \dot{y}=x^{2}-y^{2}$ \\
\hline type III(3) & $\dot{x}=x^{2}, \quad \dot{y}=x^{2}+x y$ \\
\hline type $\operatorname{III}(4)$ & $\dot{x}=0, \quad \dot{y}=x^{2}$, \\
\hline type $\operatorname{IV}(1)$ & $\dot{x}=x y, \quad \dot{y}=y^{2}$, \\
\hline type $\operatorname{IV}(2)$ & $\dot{x}=0, \quad \dot{y}=0$. \\
\hline
\end{tabular}

The parameters $\hat{p}_{1}$ and $\hat{p}_{2}$ in the canonical form of type $I \ominus$ are six-valued; i.e., two systems with $\left(\hat{p}_{1}, \hat{p}_{2}\right)$ and $\left(\hat{p}_{1}^{\prime}, \hat{p}_{2}^{\prime}\right)$ respectively are affine-equivalent if and only if the vectors $\left(\hat{p}_{1}, \hat{p}_{2}\right)$ and $\left(\hat{p}_{1}^{\prime}, \hat{p}_{2}^{\prime}\right)$ are connected with each other through a rotation by $\pm 2 \pi / 3$ and/or an inversion of the sign of $\hat{p}_{1}$.

Some fundamental invariants of a two-dimensional quadratic differential system. The following invariants and covariants known in classical invariant theory [7] are useful for our purpose.

Hessian $h^{\kappa \lambda}=\frac{1}{2} \sum_{\mu, \nu, \rho, \sigma=1}^{2} \epsilon^{\mu \nu} \epsilon^{\rho \sigma} Q_{\mu \rho}^{\kappa} Q_{\nu \sigma}^{\lambda}$, where $\epsilon^{\kappa \lambda}$ is the unit contravariant 2-vector of weight 1 with $\epsilon^{12}=-\epsilon^{21}=-1, \epsilon^{11}=\epsilon^{22}=0$.

$$
\begin{aligned}
& \text { Discriminant } D=-2 \sum_{\kappa, \lambda, \mu, \nu=1}^{2} \epsilon_{\kappa \lambda} \epsilon_{\mu \nu} h^{\kappa \mu} h^{\lambda \nu} . \\
& \text { Invariants } H=\sum_{\kappa, \lambda=1}^{2} h^{\kappa \lambda} p_{\kappa} p_{\lambda} \text { and } F=\sum_{\kappa, \lambda, \mu, \rho, \sigma=1}^{2} \epsilon^{\mu \kappa} \epsilon^{\rho \lambda} Q_{\kappa \lambda}^{\sigma} p_{\mu} p_{\rho} p_{\sigma} . \\
& \text { Covariant } T_{\kappa \lambda}=\sum_{\mu, \nu=1}^{2}\left(2 \epsilon_{\kappa \mu} \epsilon_{\lambda \nu} h^{\mu \nu}+Q_{\kappa \lambda}^{\mu} p_{\mu} / 3+p_{\kappa} p_{\lambda} / 9\right) .
\end{aligned}
$$

Given a quadratic differential system by means of these invariants and covariants we can characterize to which of the 10 types given in Theorem 7 it belongs. More precisely, 


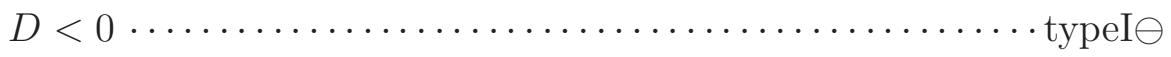

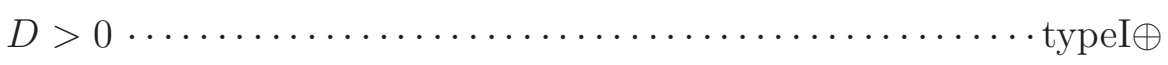

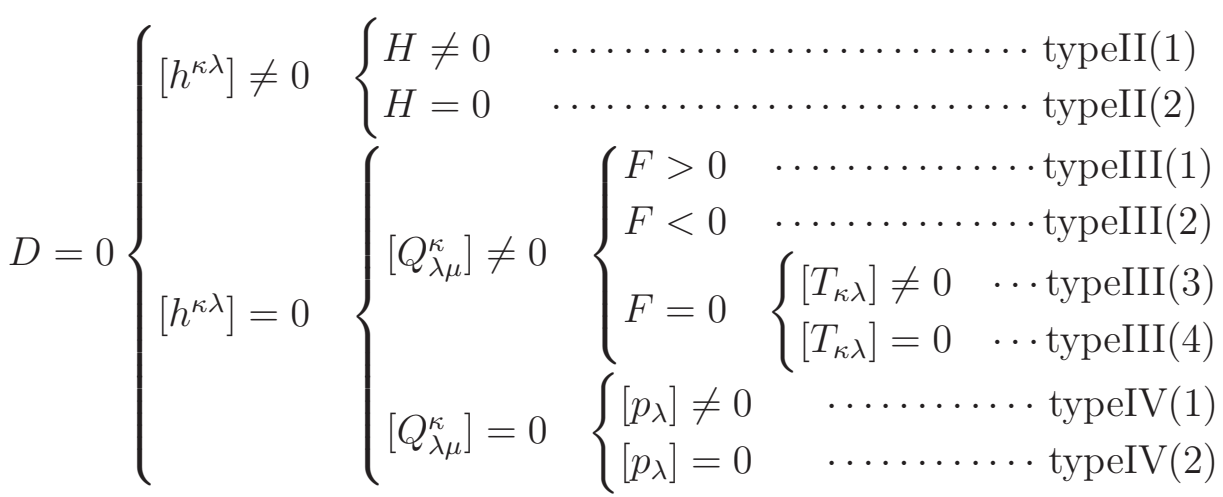

Among an infinite number of invariants of $P_{\lambda \mu}^{\kappa}$ which can be derived through combination of these fundamental invariants, the following sequence of invariants are particularly useful

$$
K_{m}=F+9(-2)^{m-3} H-27(-8)^{m-3} D, \quad m=1,2, \ldots,
$$

for more details see [4]. This sequence of invariants play a substantial role in the classification of the two-dimensional quadratic homogeneous polynomial differential systems as we show now.

The next table provides a classification of a quadratic homogeneous polynomial differential system according to the type of system that it belongs and that we shall use. In Figure 11 are shown the phase portraits that appears in this paper corresponding to the different kinds of quadratic homogeneous polynomial differential systems

\begin{tabular}{|c|c|c|c|}
\hline \multirow{6}{*}{ Type I $\ominus$} & \multicolumn{2}{|c|}{$K_{2}>0$} & Type I $\ominus(1)$ \\
\hline & \multirow[t]{2}{*}{$\overline{K_{2}<0}$} & $\overline{K_{3}>0}$ & Type I $\ominus(2)$ \\
\hline & & $\overline{K_{3}<0}$ & Type I $\ominus(3)$ \\
\hline & \multirow{3}{*}{$K_{2}=0$} & $\overline{K_{3}}>0$ & Type I $\ominus(4)$ \\
\hline & & $K_{3}<0$ & Type $\mathrm{I} \ominus(5)$ \\
\hline & & $K_{3}=0$ & Type $\mathrm{I} \ominus(6)$ \\
\hline \multirow{3}{*}{ Type $\mathrm{I} \oplus$} & \multicolumn{2}{|c|}{$K_{2}>0$ or $K_{2}=K_{3}=0$} & Type $\mathrm{I} \oplus(1)$ \\
\hline & \multicolumn{2}{|c|}{$K_{2}<0$} & Type $\mathrm{I} \oplus(2)$ \\
\hline & \multicolumn{2}{|c|}{$K_{2}=0$ or $K_{3} \neq 0$} & Type $\mathrm{I} \oplus(3)$ \\
\hline \multirow{3}{*}{ Type II(1) } & \multicolumn{2}{|c|}{$K_{2}>0$} & Type II(1-1) \\
\hline & \multicolumn{2}{|c|}{$K_{2}<0$} & Type II (1-2) \\
\hline & \multicolumn{2}{|c|}{$K_{2}=0$} & Type II(1-3) \\
\hline
\end{tabular}
described in the following table.

\section{ACKNOWLEDGMENTS}

The second author is supported by the grants MICINN/FEDER MTM 2008-03437, Generalitat de Catalunya 2009SGR410, ICREA Academia and FP7-PEOPLE-2012IRSES-316338 and 318999. The third author is supported by the grants AGAUR PIV-DGR-2010 and by FCT through the project PTDC/MAT/ 117106/2010 and through CAMGD, Lisbon. 

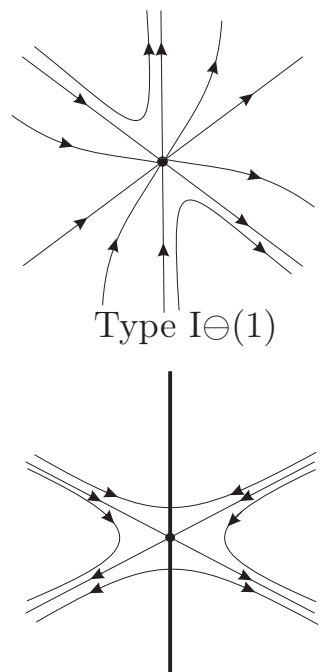

Type $\mathrm{I} \ominus(4)$

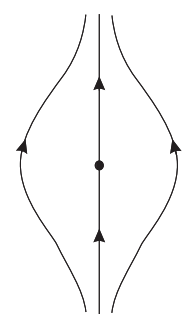

Type $\mathrm{I} \oplus(1)$

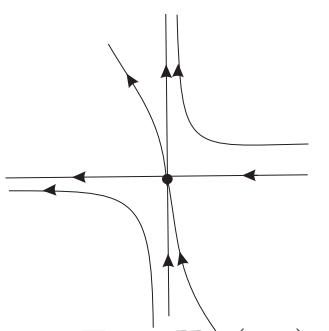

Type II $\oplus(1-1)$

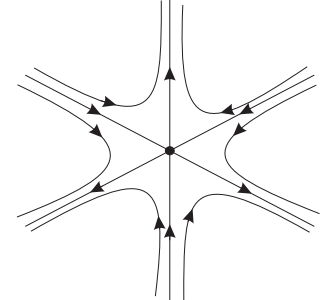

Type $\mathrm{I} \ominus(2)$

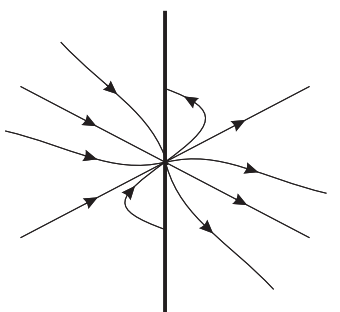

Type $\mathrm{I} \ominus(5)$

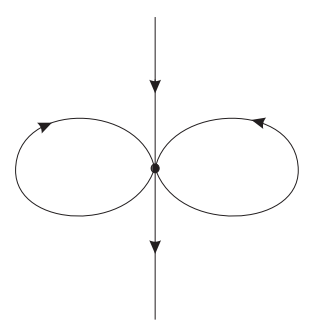

Type $\mathrm{I} \oplus(2)$

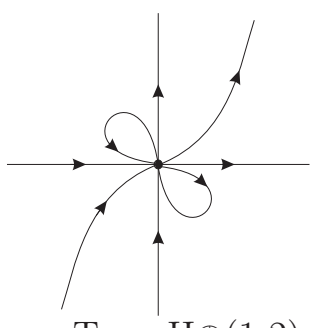

Type $\mathrm{II} \oplus(1-2)$

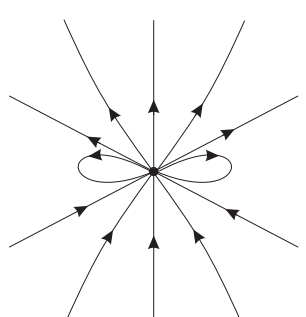

Type $\mathrm{I} \ominus(3)$

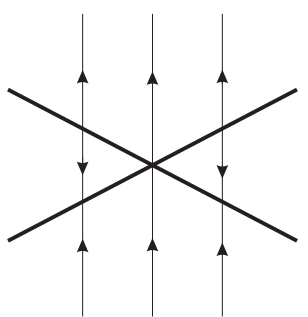

Type $\mathrm{I} \ominus(6)$

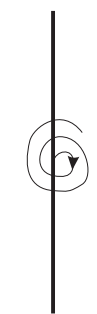

Type $\mathrm{I} \oplus(3)$

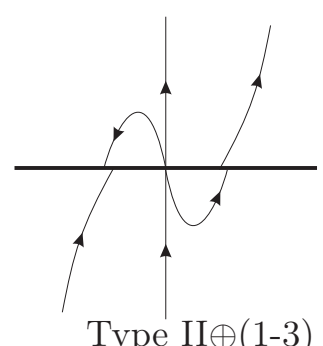

FIGURE 11. Phase portraits for quadratic homogeneous vector fields.

\section{REFERENCES}

[1] N. N. Bautin, On periodic solutions of a system of differential equations (R), Prikl. Mat. Meh. 18 (1954), 128.

[2] F. H. Busse, Transition to turbulence via the statistical limit cycle route, Synergetics, Springer Verlag, 1978, pp 39.

[3] L. Cairó, M.R. Feix And J. Llibre, Darboux method and invariants for the Lotka-Volterra and complex quadratic systems, J. Mathematical Physics 40 (1999), 2074-2091.

[4] T. Date And M. IRI, Canonical forms of real homogeneous quadratic transformations, J. Math. Anal. Appl. 56 (1976), 650-682.

[5] T. DATE, Classification and analysis of two-dimensional real homogeneous quadratic differential equation systems, J. Differential Equations 32 (1979), 311-334.

[6] F. Dumortier, J. Llibre And J.C. Artés, Qualitative theory of planar differential systems, Universitext, Springer-Verlag, 2006.

[7] E. B. Elliott, An introduction to the algebra of quantics, Oxford Univ. Press (Clarendon), London, 1913. 
[8] E. A. GonzÁLez, Generic properties of polynomial vector fields at infinity, Trans. Amer. Math. Soc. 143 (1969), 201-222.

[9] A. Kolmogorov, Sulla teoria di Volterra della lotta per l'esistenza, Giornale dell' Istituto Italiano degli Attuari 7 (1936), 74-80.

[10] G. Laval and R. Pellat, Plasma Physics, Proceedings of Summer School of Theoretical Physics, Gordon and Breach, NY, 1975.

[11] W. Li, J. Llibre, M. Nicolau and X. Zhang, On the differentiability of first integrals of two dimensional flows, Proc. Amer. Math. Soc. 130 (2002), 2079-2088.

[12] A. J. LotkA, Analytical note on certain rhythmic relations in organic systems, Proc. Natl. Acad. Sci. U.S. 6 (1920), 410-415.

[13] L. Markus, Quadratic differential equations and non-associative algebras, Annals of Mathematics Studies, Vol 45, Princeton University Press, 1960, pp 185-213.

[14] L. Markus, Global structure of ordinary differential equations in the plane: Trans. Amer. Math Soc. 76 (1954), 127-148.

[15] R. M. MAY, Stability and Complexity in Model Ecosystems, Princeton NJ, 1974.

[16] D. A. Neumann, Classification of continuous flows on 2-manifolds, Proc. Amer. Math. Soc. 48 (1975), 73-81.

[17] M. M. Peiхото, Dynamical Systems. Proccedings of a Symposium held at the University of Bahia, 389-420, Acad. Press, New York, 1973.

[18] V. Volterra, Lecons sur la Théorie Mathématique de la Lutte pour la vie, Gauthier Villars, Paris, 1931.

1 Departament de Matemàtiques, Universitat Autònoma de Barcelona, 08193 BelLATERra, BARCELONA, Spain

E-mail address: ymbolanos@gmail.com, jllibre@mat.uab.cat

2 Departamento de Matemática, Instituto Superior Técnico, Universidade Técnica De Lisboa, Av. Rovisco Pais 1049-001, Lisboa, Portugal

E-mail address: cvalls@math.ist.utl.pt 